\title{
PAPR REDUCTION FOR OFDM/OQAM SIGNALS VIA ALTERNATIVE-SIGNAL METHOD
}

\author{
Jyothi Varaprasad ${ }^{1}$, N.Padmaja ${ }^{2}$ \\ ${ }^{1}$ M.Tech. Student, Dept. of ECE, Sree Vidyanikethan Engineering College, Tirupati, Chittor (Dist), A.P \\ ${ }^{2}$ Professor of ECE Dept, Sree Vidyanikethan Engineering College, Tirupati, Chittor (Dist), A.P
}

\begin{abstract}
We deemed the PAPR reduction problem for OFDM/OQAM system. The PAPR reduction is the serious problem for implementations of both OFDM and OFDM/OQAM systems due to their high PAPR. The OFDM/OQAM signal is generated by summing over M time-shifted OFDM/OQAM symbols, where the successive symbols are interdependent with each other. The AS (Alternative-Signal) method directly leads to the independent AS (AS-I) and joint AS (AS-J) algorithms. The AS-I algorithm reduces the PAPR symbol by symbol with low complexity and AS-J applies optimal joint PAPR reduction among $M$ OFDM/OQAM symbols with much higher complexity. A sequential optimization procedure denoted AS-S have been proposed to balance the computation complexity and system performance in this paper. AS-S algorithm, which adopts a sequential optimization procedure over time with computational complexity linearly increasing with M. The Simulation results have been provided for performance comparison of $A S-I, A S-J$, and $A S-S$ algorithms.
\end{abstract}

Keywords-Peak-to-Average power ratio (PAPR), orthogonal frequency division multiplexing with offset quadrature amplitude modulation (OFDM/OQAM), Alternative-signal(AS), cyclic prefix $(C P)$.

\section{INTRODUCTION}

The multicarrier modulation technique for LTE-A (Long term Evolution Advanced) and other wireless standards, OFDM/OQAM was used because of its high spectrum efficiency [1]-[3]. The OFDM/OQAM signal can be obtained by summing over $\mathrm{M}$ time-shifted OFDM/OQAM symbols, each of which is obtained by letting N QAM symbols pass through a prototype filter [4] and they are modulated with $\mathrm{N}$ orthogonal subcarriers. The OFDM/OQAM systems have some advantages compared with the traditional OFDM systems, viz. The $\mathrm{CP}$ is no longer required and the side lobe of its power spectral density is very low [4]. The disadvantages of OFDM/OQAM are the heavier computation cost because of the extra filtering operations and more complex channel equalization.

For both the OFDM and OFDM/OQAM systems, the main issues for implementations are their relatively high PAPR. For OFDM systems several methods such as companding [6], clipping [7], PTS (Partial Transmit Sequence)[8] and SLM(Selective Mapping) [9] have been proposed. For instance, the companding scheme compacted the huge samples and extended the small samples. The clipping scheme limited the OFDM signal to reduce the PAPR at a given amplitude threshold. The SLM and PTS methods generated several OFDM symbols by multiplying the original symbol with different phase rotation vectors, and chose one with the lowest PAPR for transmission. For OFDM/OQAM systems, the clipping scheme [10]-[11], the SLM method [12], and the precoding scheme have been employed for PAPR reduction. In the clipping scheme was introduced to the PTS and SLM e OFDM/OQAM systems, and it increased the bit error rate (BER) and distended the side lobe. To avoid the BER increasing, an iterative noise cancelation technique called Buss gang noise cancelation was applied at the receiver [10], but it may increase the decoding complexity at the receiver. In the clipping-based schemes (including the tone reservation and active constellation extension) were employed to reduce the PAPR of OFDM/OQAM signals.

The SLM method is applied to divided each OFDM/OQAM symbol into two parts (real and imaginary), randomly adopted the phase rotation vectors for the first $2 K$ parts, with $K$ being the parameter associated with the length of the prototype filter, and then optimized the $(2 K+1)^{\text {th }}$ part to reduce the PAPR. Due to the random choice of the phase rotation vectors, if the prototype filter length is long then the PAPR reduction performance is poor. In the single-carrier OFDM/OQAM system a precoding matrix was inserted between the multiplexer and the OFDM/OQAM modulator to reduce the PAPR.

\section{OFDM/OQAM SYSTEM MODEL}

The OFDM/OQAM transmitter structure is shown in Fig.1, it contains $\mathrm{N}$ subcarriers. After QAM modulation, the input QAM symbols are first serial to parallel converted to data matrix $\mathrm{X}$ is given by

$$
X=\left[X^{0}, X^{1}, \ldots, X^{M-1}\right]
$$




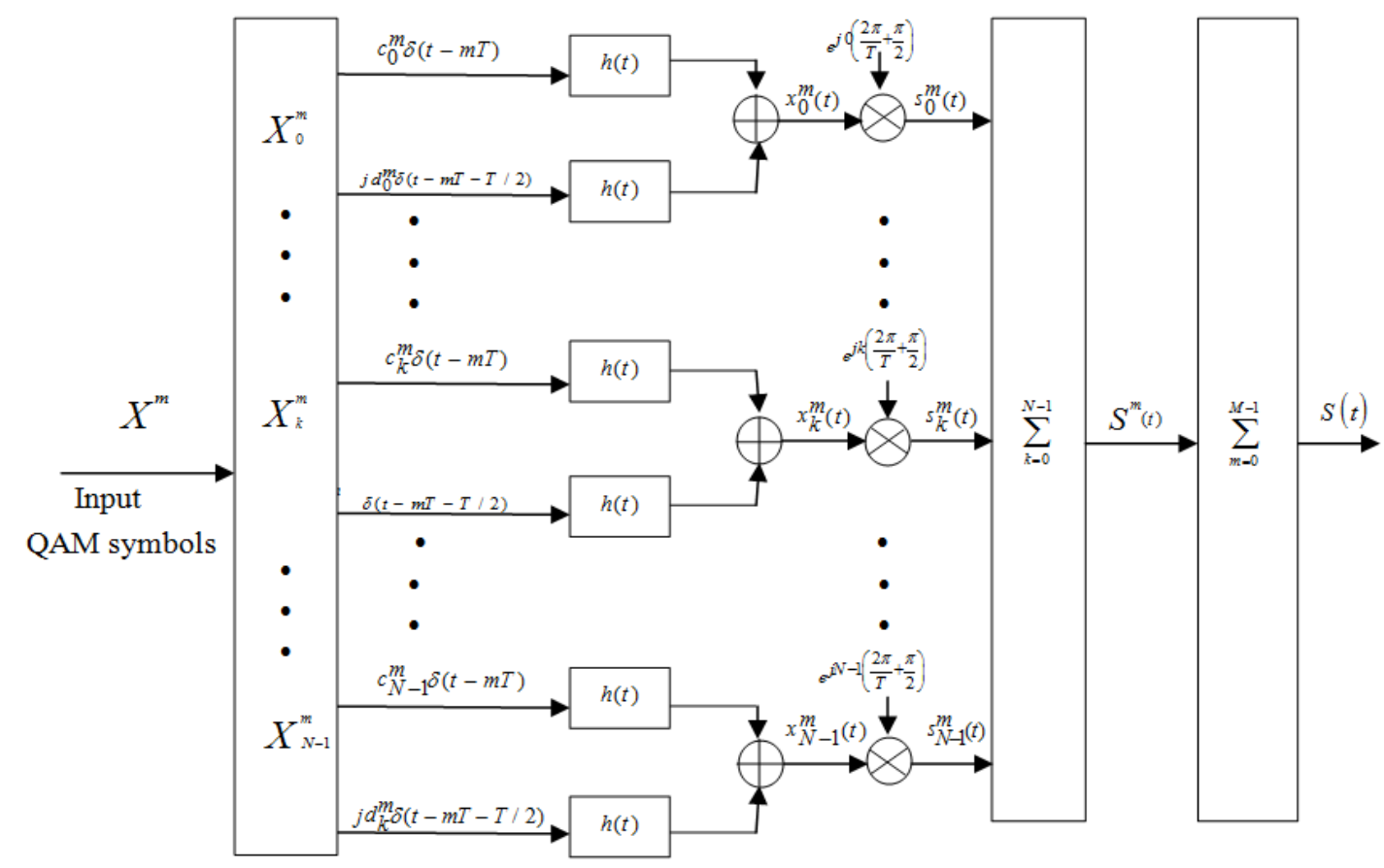

Fig.1. Typical OFDM/OQAM transmitter

Where $\mathrm{M}$ is the number of data blocks, and $X^{m}$ is the $m^{\text {th }}$ data block which is given by

$$
X^{m}=\left[X_{0}^{m}, X_{1}^{m}, \ldots, X_{N-1}^{m}\right]^{T}
$$

Where $T$ is the Transpose.

The $m^{\text {th }} \mathrm{QAM}$ symbol on the $k^{\text {th }}$ subcarrier is given by

$$
X_{k}^{m}=c_{k}^{m}+j d_{k}^{m}
$$

Where $c_{k}^{m}$ and $d_{k}^{m}$ are the real and imaginary parts of $x_{k}^{m}$, respectively.

The real and imaginary parts of $X_{k}^{m}$ are passed through the prototype filter to obtain

$$
x_{k}^{m}(t)=c_{k}^{m} h(t-m T)+j d_{k}^{m} h\left(t-\frac{T}{2}-m T\right)
$$

Where $\mathrm{T}$ is the symbol period and $h(t)$ is the response of the prototype filter. Length of $h(t)$ is KT; where $\mathrm{K}$ is even integer [4] and we adopt $\mathrm{K}=4$ in this paper.

Then, $x_{k}^{m}(t), \quad k=0,1, \ldots ., N-1 \quad$ are modulated with $N$ orthogonal subcarriers to obtain

$$
\begin{gathered}
s_{k}^{m}(t)=\left\{c_{k}^{m} h(t-m T)+j d_{k}^{m} h\left(t-\frac{T}{2}-m T\right)\right\} e^{j k\left(\frac{2 \pi}{T} k+\frac{\pi}{2}\right)} \\
k=0,1 \ldots N-1
\end{gathered}
$$

Add all the $s_{k}^{m}(t)$ of $N$ subcarriers together, we obtain the $m t h$ OFDM/OQAM symbol $s^{m}(t)$ as

$$
\boldsymbol{S}^{m}(t)=\sum_{k=0}^{N-1} s_{k}^{m}(t), \quad m T \leq t \leq\left(m+k+\frac{1}{2}\right) T
$$

Note that the length of each OFDM/OQAM symbol $s^{m}(t)$ is

$$
(k+1 / 2) T \text {. }
$$

Finally, the desired OFDM/OQAM signal $s(t)$ is obtained by summing over M OFDM/OQAM symbols, i.e.,

$$
s(t)=\sum_{m=0}^{M-1} S^{m}(t), \quad 0 \leq t \leq\left(M+k-\frac{1}{2}\right) T
$$

The structure of OFDM/OQAM signal is shown in fig.2, Where,

The symbol rate $=1 / \mathrm{T}$ and

The length of each OFDM/OQAM symbol $=(K+1 / 2) T$

The length of OFDM/OQAM signal $=\left(M+K-\frac{1}{2}\right) T$ 


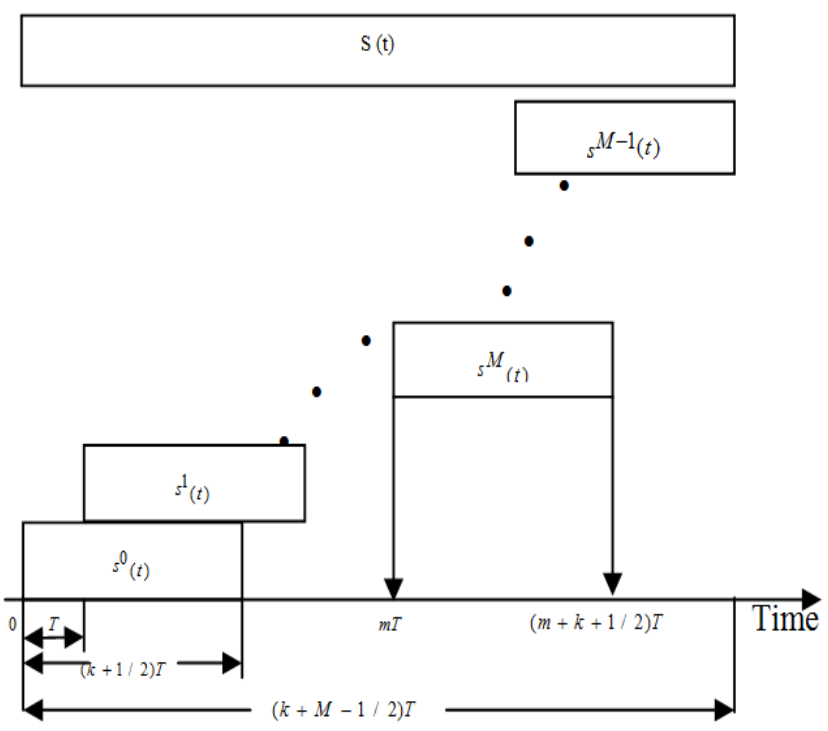

Fig 2 Structure of OFDM/OQAM signal

In the conventional OFDM systems, the OFDM symbol can be obtained by taking the IFFT operation over the $N$ QAM symbols, and the length of each OFDM symbol is $T$. Since the OFDM symbol rate is $1 / T$, there is no overlap between any adjacent OFDM symbols, and the PAPR of each OFDM symbol is defined as the ratio of the peak power to the average power. Due to the special signal structure, the PAPR definition for the proposed OFDM/OQAM systems needs to be modified.

OFDM/OQAM signal $s(t)$ is first divided into $M+K$ intervals, each of which is with length $T$ (except the last one, with length $T / 2)$. Then, the PAPR for $s(t)$ in the $p$ th interval is

$$
\begin{aligned}
& \xi^{p}=10 \log _{10} \frac{\max _{p T \leq t \leq(p+1) T}|s(t)|^{2}}{p_{\text {avg }}}, p=0,1, \ldots, M+K-2 \\
& \xi^{p}=10 \log _{10} \frac{\max _{p T \leq t \leq(p+1 / 2) T}|s(t)|^{2}}{p_{\text {avg }}}, p=0,1, \ldots, M+K-1
\end{aligned}
$$

Where $p_{\text {avg }}$ is the average power of $s(t)$.

The notifications that are used in above equations may defined in below

- $\quad X$ is the input data matrix and

- $\quad X^{m}$ is the $m^{\text {th }}$ data block.

- $\quad X_{k}^{m}$ is the $m^{\text {th }} \mathrm{QAM}$ symbol on the $k^{\text {th }}$ subcarrier.

- $S^{m}(t)$ is the $m^{\text {th }}$ OFDM/OQAM symbol.

- $s(t)$ is the OFDM/OQAM signal.

\section{ALTERNATIVE-SIGNAL METHOD}

\subsection{AS-I Algorithm}

From the SLM method, the AS-I algorithm reduces the PAPR by optimally choosing one phase rotation vector from a given set for each OFDM/OQAM symbol. Over different OFDM/OQAM symbols, the phase rotation vectors may be different. Denote the set of candidate phase rotation vectors as

$$
B=\left\{b^{0}, b^{1}, \ldots . ., b^{U-1}\right\}
$$

Where $U$ is the size of $\mathrm{B}$, and $b^{u}, 0 \leq u \leq U-1$, is the phase $u^{\text {th }}$ rotation vector, which is defined as

$$
b^{u}=\left[b_{0}^{u}, b_{1}^{u}, \ldots \ldots, b_{N-1}^{u}\right]^{T}
$$

With $b_{k}^{u}=e^{(2 \pi i / W)}, i=0,1, \ldots W-1[11]$. We adopted $W=2$ for simplicity in this paper. $b^{m, u}=\left[b_{0}^{m, u}, b_{1}^{m, u}, \ldots \ldots, b_{N-1}^{m, u},\right]^{T}$ is the phase rotation vector used by the $m^{\text {th }}$ OFDM/OQAM symbol $S^{m}(t)$. Usually, $B$ is assumed to be known at both the transmitter and the receiver.

After $s_{k}^{m}(t)$ is generated in [6], AS-I generates $\tilde{s}_{k}^{m}(\mathrm{t})$ by multiplying the corresponding element in the selected phase rotation vector i.e.,

$$
\tilde{s}_{k}^{m}(\mathrm{t})=s_{k}^{m}(\mathrm{t}) b_{k}^{m, u}
$$

Then, the new OFDM/OQAM symbol $\tilde{s}_{k}^{m}(\mathrm{t})$ is expressed as

$$
\tilde{s}_{k}^{m}(\mathrm{t})=\sum_{k=0}^{N-1} s_{k}^{m}(\mathrm{t}) b_{k}^{m, u}
$$

Thus, the PAPR reduction problem with the AS-I algorithm for the $m^{\text {th }}$ OFDM/OQAM symbol $s^{m}(t), m=0,1 ., M-1$, can be formulated as

$$
\text { (P1): } \quad \min _{b^{m, u}} \max _{m T \leq t \leq\left(m+K+\frac{1}{2}\right) T}\left|\sum_{k=0}^{N-1} s_{k}^{m}(t) b_{k}^{m, u}\right|^{2}
$$

Subject to: $b^{m, u} \in B$.

Note that we adopt the peak power as the design metric throughout this paper. This is because the PAPR reduction should come from the peak power reduction rather than the average power increasing. Given the finite dimensionality of $B$, exhaustive search is adopted here to search the optimal $b^{m, u}$. For each $s^{m}(t)$ the complexity of searching the optimal $b^{m, u}$ is on the order of $O(U)$, i.e., for each $S^{m}(t)$, we 
take $U$ searches. Thus, the complexity for all $S^{m}(t), \mathrm{m}=$ $0,1 \ldots \mathrm{M}-1$ is on the order of $O(U M)$.

Remark 1: After obtaining the PAPR reduced OFDM/OQAM signal ^ $s(t)$, the transmitter should send side information to the receiver about which phase rotation vector is selected for

$$
S^{m}(t), m=0,1 \ldots, M-1 .
$$

The PAPR reduction performance achieved by the AS-I algorithm is given in Section IV. The AS-I algorithm does not perform well enough since it ignores the structure of the OFDM/OQAM signals, i.e., the correlation among adjacent OFDM/OQAM symbols, where as reducing the PAPR of $S^{m}(t)$ independently is strictly suboptimal. The AS-J algorithm is proposed to develop the PAPR reduction performance in the inter symbol correlations.

\subsection{AS-J Algorithm}

First, The AS-J algorithm chooses one phase rotation vector from the given $B$ for each OFDM/OQAM symbol $S^{m}(t)$; then, it applies a joint PAPR reduction scheme among all the M OFDM/OQAM symbols.

Similarly, after $\tilde{s}_{k}^{m}(\mathrm{t})$ is generated, as we did in the AS-I algorithm, the PAPR reduction problem could be formulated as

$$
\text { (P2): } \min _{b^{0, u}, \ldots, b^{M-1, u}} \max _{0 \leq t \leq\left(m+K-\frac{1}{2}\right) T}\left|\sum_{m=0}^{M-1} \sum_{k=0}^{N-1} s_{k}^{m}(t) b_{k}^{m, u}\right|^{2}
$$

Subject to: $\quad b^{m, u} \in B . \quad m=0,1, \ldots . . M-1$.

It is clearly shown, the AS-I algorithm is simple but performs badly but the AS-J algorithm performs well but bears high complexity. To balance the PAPR reduction performance and the complexity, the AS-S algorithm is proposed in the following.

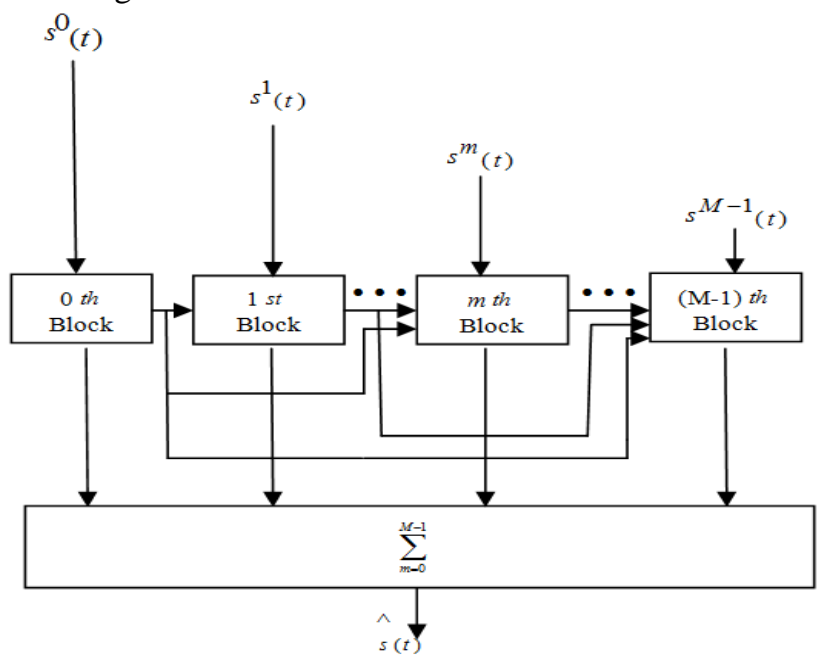

Fig.3. Structure of the AS-S algorithm.

\subsection{AS-S Algorithm}

The main idea of the AS-S algorithm is shown in Fig. 3. AS$\mathrm{S}$ algorithm adopts a sequential optimization procedure. In the $m^{\text {th }}$ block, by taking into account the previous OFDM/OQAM symbols, i.e., $s^{0}(t), s^{1}(t), \ldots \ldots . ., s^{M-1}(t)$, we reduce the peak power of $s^{m}(t)$. The procedure of the AS$\mathrm{S}$ algorithm is described as follows.

In the 0 -th block, we multiply $s^{0}(t)$ by different phase rotation vectors and choose the one with the minimum peak power, which is denoted as $\hat{s} s^{0}(t)$. then, $\hat{s} s^{0}(t)$ is sent to the first block to solve the following problem:

$$
\min _{b^{1, u}} \max _{2 T \leq t \leq 4 T}\left|s^{0}(t)+\sum_{k=0}^{N-1} s_{k}^{1}(t) b_{k}^{1, u}\right|^{2}
$$

Subject to: $b^{m, u} \in B$.

$b^{1, u}$ is the optimal phase rotation vector and the new generated symbol is cast as

$$
\wedge^{1}(t)=\sum_{k=0}^{N-1} s_{k}^{1}(t) b_{k}^{1, u}
$$

Next, $\wedge s^{1}(t)$ and $\hat{s} s^{0}(t)$ are both sent to the second block to calculate the new symbol $\wedge^{2}(t)$. We repeat the given procedure until the $(M-1)^{\text {th }}$ block.

AS-S is a sequential optimization procedure. Specifically, in the $m^{\text {th }}$ block, $\mathrm{m}=1,2 \ldots \mathrm{M}-1$, the optimization problem could be cast as follows

$$
\text { (P3): } \min _{b^{m, u}} \max _{(m+1) T \leq t \leq(m+\Gamma) T}\left|\sum_{t=0}^{m-1} \hat{s} s^{1}(t)+\sum_{k=0}^{N-1} s_{k}^{m}(t) b_{k}^{m, u}\right|^{2}
$$

Subject to: $b^{m, u} \in B$.

Note that $\Gamma$ is a key parameter that significantly affects the PAPR reduction performance and will be discussed in Remark 2. In Problem (P3), the search complexity for each symbol $S^{m}(t)$ is on the order of $O(U)$, and the complexity for all the $M$ symbols is on the order of $O(U M)$. Similarly, the number of bits to transmit the side information is also equal to $M \operatorname{Mog}_{2}(U)$.

Remark 2: We plot the amplitudes of $h(t-m T)$ and $S^{m}(t)$ in Fig. 4, where the parameters of the prototype filter are the same as those in [4]. It can be seen that the large-amplitude samples of $h(t-m T)$ are located within $\{(m+K / 2-1 / 2) T \leq t$ $\leq(m+K / 2+1 / 2) T\}$. For $\mathrm{h}(\mathrm{t}-\mathrm{mT}-\mathrm{T} / 2)$, its large-amplitude samples are located within $\{(\mathrm{m}+\mathrm{K} / 2) \mathrm{T} \leq \mathrm{t} \leq(\mathrm{m}+\mathrm{K} / 2+$ 1)T . According to (5) and (6), we could obtain that the large-amplitude samples of $s^{m}(t)$ are located within $\{(\mathrm{m}+\mathrm{K} / 2-1 / 2) \mathrm{T} \leq \mathrm{t} \leq(\mathrm{m}+\mathrm{K} / 2+1) \mathrm{T}\}$. Intuitively, to obtain a good PAPR reduction performance, the large-amplitude samples of $s^{m}(t)$ should be included in the optimization duration $\{(\mathrm{m}+1) \mathrm{T} \leq \mathrm{t} \leq(\mathrm{m}+\Gamma) \mathrm{T}\}$ in Problem $(\mathrm{P} 3)$, i.e., $\Gamma$ should satisfy $\Gamma \geq K / 2+1$. Furthermore, since $s^{m}(t)$ only 
spans over $\{\mathrm{mT} \leq \mathrm{t} \leq(\mathrm{m}+\mathrm{K}+1 / 2) \mathrm{T}\}$, it follows that $\Gamma \leq$ $(\mathrm{K}+1 / 2)$. Thus, we conclude that $(\mathrm{K} / 2+1) \leq \Gamma \leq(\mathrm{K}+1 / 2)$ is a good choice.

The AS-S algorithm is summarized as follows.

Step 1: Initialization: $m=1$. Multiply $s^{0}(\mathrm{t})$ with different Phase rotation vectors and chooses one with minimum Peak power $\tilde{s}^{0}(\mathrm{t})$ and then sent to the first block.

Step 2: Solve (P3) in the $m^{\text {th }}$ block, and the new symbol is denoted as $\hat{s}^{m}(\mathrm{t})$.send $\hat{s}^{0}(\mathrm{t}), \hat{s}^{1}(\mathrm{t}), \ldots, \hat{s}^{m}(\mathrm{t})$ to the next block.

Step 3: Set $m=m+1$, if $m=M-1$, go to step 2; otherwise, Calculate $\hat{s}(\mathrm{t})=\sum_{M=0}^{M-1} \hat{s}^{m}(\mathrm{t})$ and calculate the output.
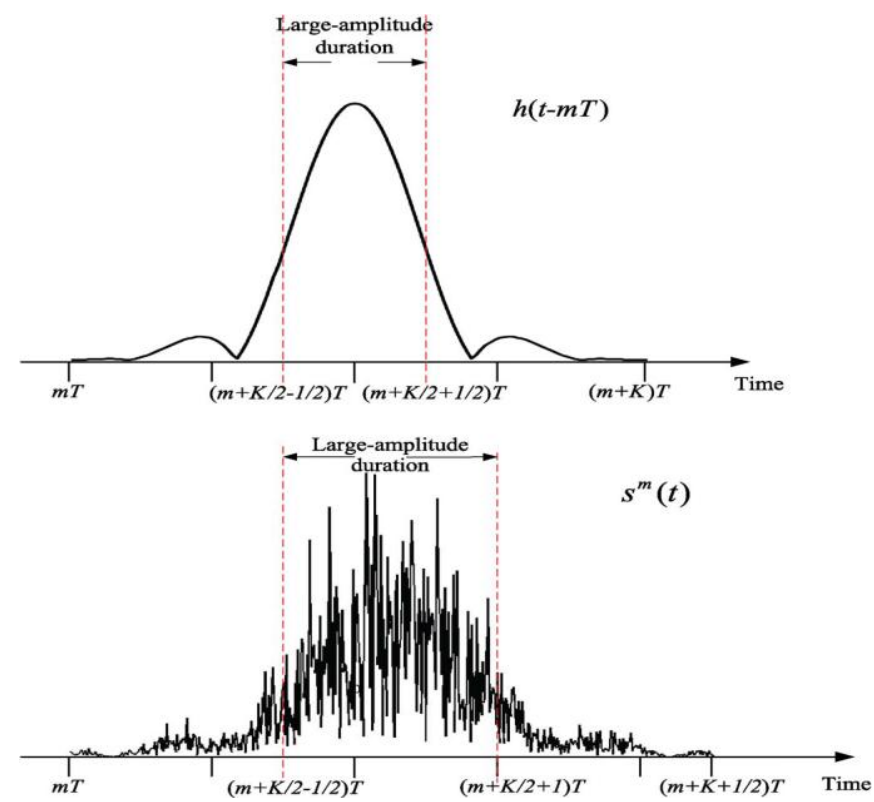

Fig.4 Amplitudes of $h(t-m T)$ and $s^{m}(t)$, respectively

\section{SIMULATION RESULTS}

Here, simulations were done to compute the PAPR reduction performance of AS-S algorithm. In all simulations, the overlapping factor is taken as $L=4$. The CCDF is used to calculate PAPR performance ad it is described as the probability that the PAPR $\xi$ increased a given threshold $P A P R_{0}$, i.e.,

$$
P\left(P A P R_{0}\right)=\operatorname{Pr}\left[\xi \geq P A P R_{0}\right]
$$

Where The $p_{\text {avg }}$ of PAPR-reduced signal $\hat{s}(\mathrm{t})$ is slightly increase compared with $s(\mathrm{t})$.

All CCDF curves for AS algorithm plotted in Fig.5. Where 4-QAM is applied and choose $N=32, M=4, U=8$, and $\Gamma=4$. We set $M=4$ to obtain AS-J curve and also plotted OSLM method for comparison purpose. It is clearly identified that the PAPR Could be reduced about by $1.8 \mathrm{~dB}$ with OSLM, only $1.5 \mathrm{~dB}$ with $\mathrm{AS}-\mathrm{I}$ and almost $3.8 \mathrm{~dB}$ with $\mathrm{AS}-\mathrm{S}$ while compared with the original OFDM/OQAM signal.

In Fig. 6, we shows the performance of AS-I ad AS-S with different values of $U$. where 16-QAM is taken, $M=10^{3}$, $N=128, \Gamma=4$ and $U$ is selected as $U=4,16,64,128$.

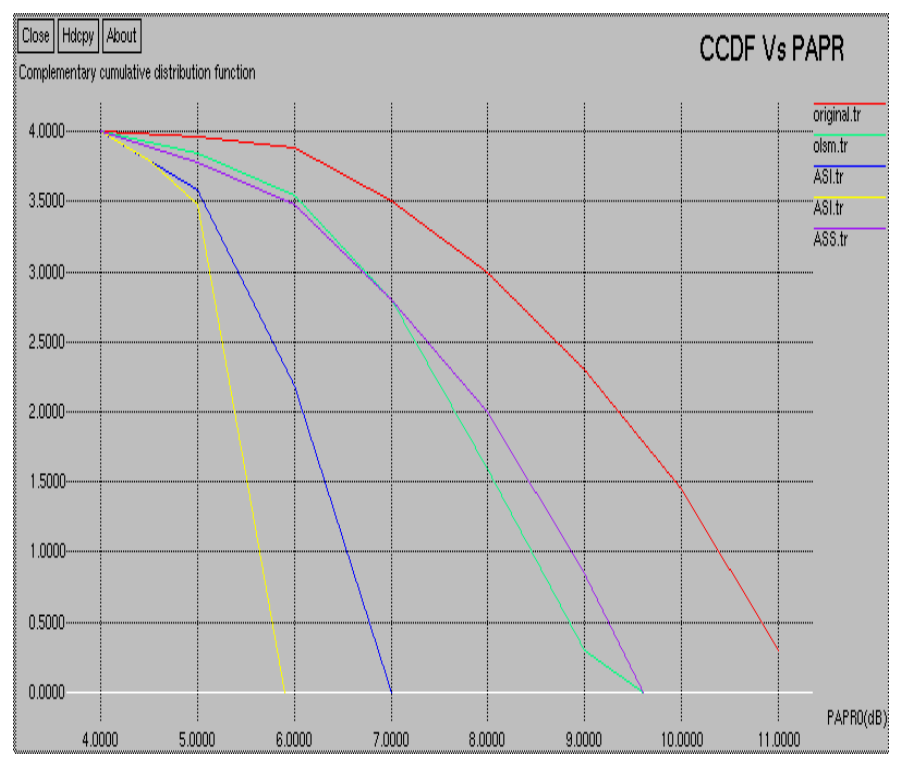

Fig.5. CCDF versus $P A P R_{0}$ for different schemes, 4-QAM,

$$
\mathrm{N}=32, \mathrm{M}=4, \mathrm{U}=8 \text {, and } \Gamma=4 \text {. }
$$

Where $M=1000$ is a extremely large number, so we do not plot the AS-J curves. Since, compared with the original signal AS-S could give the PAPR reduction gains of $2.8 \mathrm{~dB}$, $4 \mathrm{~dB}, 4.5 \mathrm{~dB}$, and $4,7 \mathrm{~dB}$ when $U=4,16,64,128$, respectively. Hence increasing $U$ can improve the PAPR reduction performance for AS-S. However, $U$ increases, the PAPR reduction performance scantily improves AS-I.

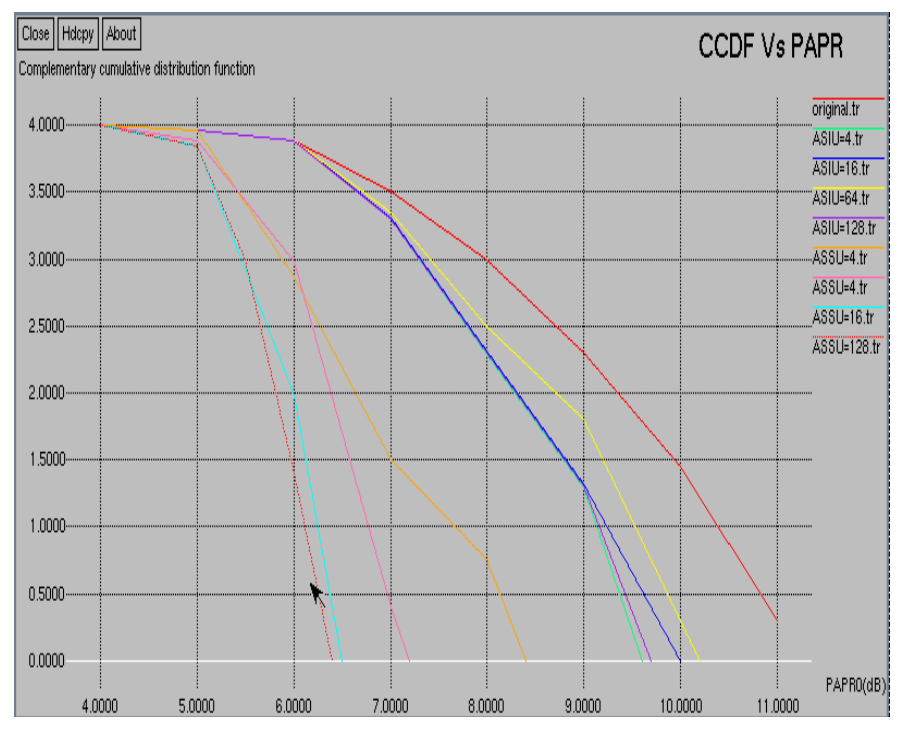

Fig. 6.CCDF versus $P A P R_{0}$ for $\mathrm{AS}-\mathrm{I}$ and $\mathrm{AS}-\mathrm{S}$ with different $U$, 16-QAM, $M=10^{3}, N=128, \Gamma=4$. 
We shown in Remark 2, $\Gamma$ is a key parameter for the PAPR reduction performance with AS-S. The Fig.7 describes the CCDF curves for AS-S with distinct values of $\Gamma$, 16-QAM is used, $M=10^{3}, N=128, U=32$. From the plot, it cleared that the PAPR reduction performance with $\Gamma=2$ is much worse with $\Gamma=3$. The AS-S provides about 0.3 -dB PAPR reduction when $\Gamma=2$, but AS-S provides about 4.4-dB PAPR reduction with $\Gamma=3$. When set $\Gamma=2$ in problem (P3), the optimization duration is $\{(m+1) T \leq t \leq(m+$ 2) $T\}$ and hence the large amplitude samples of $s^{m}(\mathrm{t})$ are positioned within $\{(m+3 / 2) T \leq t \leq(m+3) T\}$, so they can't be minimized while solving problem (P3), it may cause poor PAPR reduction performance. When $\Gamma=3$, the high amplitude samples are placed within the optimization duration and hence good PAPR reduction performance is achieved.

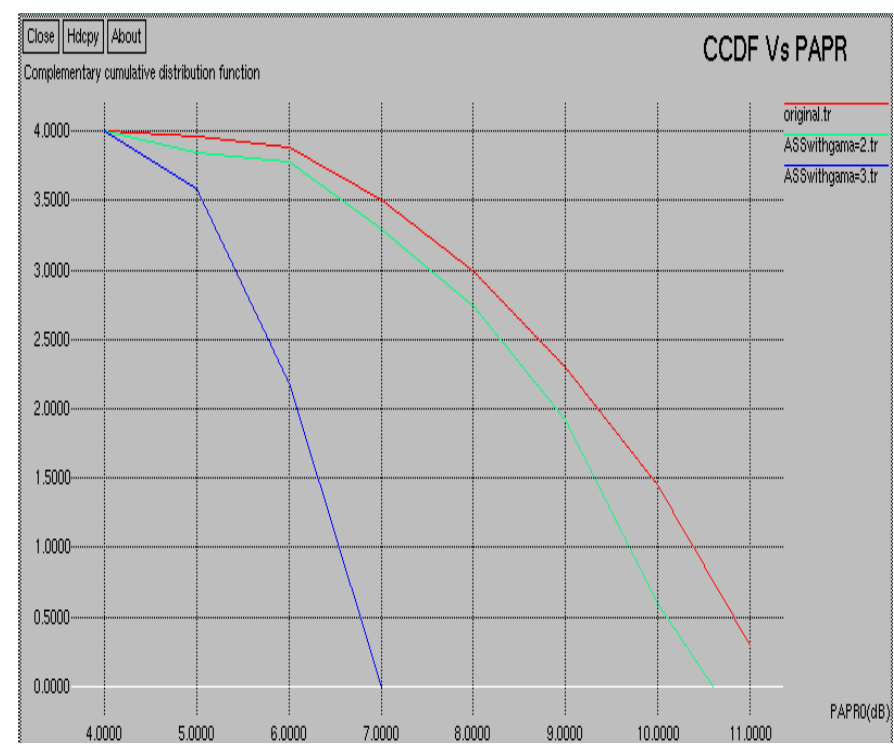

Fig. 7. CCDF versus $P A P R_{0}$ for $\mathrm{AS}-\mathrm{S}$ with different $\Gamma$, 16-

$$
\text { QAM, } M=10^{3}, N=128, U=32 \text {. }
$$

\section{CONCLUSION}

The Alternative -Signal method includes the AS-I, AS-J, and AS-S algorithms, for the PAPR reduction of the OFDM/OQAM signals. The AS-I, AS-J, and AS-S methods require the same amount of side information i.e., which phase rotation vector was used at the transmitter to send OFDM/OQAM symbol. The computational complexities of AS-I and AS-S are of the order of $O\left(U^{M}\right)$ where $U$ is the number of the phase rotation vectors and $M$ is the number of the OFDM/OQAM symbols, which was much lesser than that of AS-J. Simulation results describes that the AS-S algorithm is a preferred one since it gives a much better PAPR reduction performance than the AS-I algorithm and we catch much lower computational complexity than the AS-J algorithm.

\section{REFFERECES}

[1] Yang Zhou, Tao Jiang, Senior member, IEEE, Chuan- Huang, Member, IEEE, and Shuguang Cui, senior-Member, IEEE," Peak-to-Average power Ratio Reduction for OFDM/OQAM Signals via Alternative-Signal Method," IEEE Trans., Vehicular Technology, vol.63, no.1, January 2014.

[2] Physical Layer for Dynamic Spectrum Access and Cognitive Radio, An European Project, i.e., Phydyas project, D5.1 and D8.1. [Online]. Available: http://www.ict-phyyas.org.

[3] B.Farhang-Boroujeny, "OFDM versus filter bank multi- carrier," IEEE, Signal Process, Mag., vol.28, no.3, pp.92-112, May 2011.

[4] P. Siohan, C. Siclet, and N. Lacaille," Analysis and Design of OFDM/OQAM systems based on filter bank theory," IEEE Trans. Signal Process, vol. 50, no.5, pp.1170-1183, May 2002.

[5] J. Tellado," Peak-to-average power reduction for multi- carrier modulation," Ph.D, dissertation, Stanford univ., CA, USA, Sep, 1999.

[6] T. Jiang, W. Xiang, P. C. Richardson, D. Qu, and G. $\mathrm{Zhu}$, "On the nonlinear companding transform for reduction in PAPR of MCM signals," IEEE Trans., Wireless Commun., vol. 6, no. 6, pp. 2017-2021, Jun, 2007.

[7] J. Armstrong, "Peak-to-average power reduction for OFDM by repeated clipping and frequency domain filtering," Electron. Lett, vol. 38, no. 5,pp. 246-247, Feb. 2002.

[8] S. H. Muller and J. B. Huber, "OFDM with reduced Peak-to-average power ratio by optimum combination of partial transmit sequences, "Electron. Lett, vol. 33, no.5, pp. 368-369, Feb. 1997.

[9] S. Goff, B. Khoo, C. Tsimenidis, and B. Sharif, "A novel selected mapping technique for PAPR reduction in OFDM systems," IEEE Trans., Commun., vol. 56, no.11, pp.1775-1779, Nov. 2008.

[10] Z. Kollar and P. Horvath, "PAPR reduction of FBMC by clipping and its iterative compensation," J. Comput. Netw. Commun, vol. 2012, pp. 1-11, Feb. 2012.

[11] Z. Kollar, L. Varga, and K. Czimer, "Clipping-based iterative PAPR reduction techniques for FBMC," in Proc. InOWo, Essen, Germany, Aug. 2012, pp. 1-12.

[12] A. Skrzypczak, J. Javaudin, and P. Sinhan, "Reduction of the peak-to-average power ratio for the OFDM/OQAM modulation," in Proc. IEEE VTC, Melbourne, Vic., Australia, May 2006, pp. 20182022. 


\section{BIOGRAPHIES}

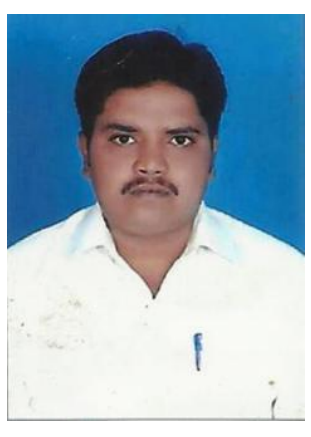

$\mathrm{J}$ Varaprasad is currently perusing his M.Tech (Communication Systems) in

Sree Vidyanikethan Engineering College, Tirupati, and A.P. He received his B.Tech degree in Electronics and Communication Engineering from Visvodaya Institute of Technology and Science, Kavali, Jawaharlal Nehru Technological University Hyderabad, Hyderabad, A.P.

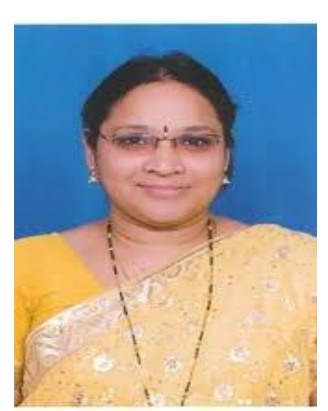

Dr. Nimmagadda Padmaja received B E (ECE) from University of Mumbai, India in 1998 and M Tech (Communication Systems) from $\mathrm{S}$ $\mathrm{V}$ University College of Engineering, Tirupati in 2003 and Ph.D from $\mathrm{S} V$ University in the area of Atmospheric Radar Signal Processing. Currently she is working as Professor, Sree Vidyanikethan Engineering College (Autonomous), Tirupati. India. She has 16 years of teaching experience. She stood FIRST in M.Tech (Electronic Instrumentation and Communication systems) Degree Examination of $\mathrm{S} \mathrm{V}$ University in the year 2003. She has published 22 technical papers in various in International Journals \& conferences. She is Life Member of ISTE, IETE and IACSIT. She is the Principal -Investigator of on-going Research Project funded by ISRO worth 15 Lakhs in the area of "MST Radar Signal Processing'. Presently she is serving as Executive Council Member for IETE local Centre, Tirupati, Member, Board of Studies, ECE Department, and Sree Vidyanikethan Engineering College. She is Member, Review Panel of various International Journals. She organized several National and International Workshop, Conferences and FDPs. She served as Head, Department of ECE, CREC, Tirupati and also Received Best Teacher Award for the year 2007-2008 in C R Engineering College. 